\title{
SwissDRG: ein Jahr vor der Einführung
}

\section{Beatrix Meyer}

Leiterin Tarifdienst FMH und Bereich SwissDRG

La version française de cette article paraîtra dans le prochain numéro du Bulletin des médecins suisses.

Vor kurzem hat die SwissDRG AG auf die Forderungen und Auflagen geantwortet, die der Bundesrat im Zusammenhang mit der Einführung des Fallpauschalensystems SwissDRG per 1. Januar 2012 gestellt hatte. Im Zentrum der Antwort stehen dabei die Verbesserung der Datenqualität der Spitäler, der Einbezug der Anlagenutzungskosten in das SwissDRG-System, das Aufzeigen des weiteren Vorgehens bezüglich der Tarifmodelle Psychiatrie und Rehabilitation und die Qualitätssicherung. Der folgende Beitrag erläutert diese Themen und geht auf weitere aktuelle und für die Ärzteschaft relevante Aspekte, zum Beispiel die Zusatzentgelte, ein.

\section{Datenerhebung durch alle Spitäler}

Heute werden die Leistungsdaten durch alle Spitäler erhoben, die Kostendaten bilden hingegen nur 46 Netzwerkspitäler ab. Unter Berücksichtigung einer Übergangsfrist und der möglichen Auflagen der Kantone strebt die SwissDRG AG an, die Erhebung der Kostendaten auf alle Spitäler auszuweiten. Ziel ist es, die Datenbasis der Spitäler mittelfristig zu erweitern und qualitativ zu verbessern. Bis im Jahr 2014 (SwissDRG-Version 3.0) möchte die SwissDRG AG die Kostengewichte vollständig anhand von Schweizer Daten berechnen können - und nicht mehr auf (helvetisierte) deutsche Kostengewichte zurückgreifen müssen.

\section{Die FMH setzt sich seit langem dafür ein, dass im} SwissDRG-System Zusatzentgelte etabliert werden.

Korrespondenz: Beatrix Meyer Tarifdienst FMH Froburgstrasse 15 CH-4600 Olten Tel. 0622879696 swissdrg@fmh.ch
Seit dem 1. Januar 2011 lassen sich die Leistungsdaten differenzierter kodieren, denn 89\% der knapp 1800 CHOP-Anträge aus den Antragsjahren 2008/ 2009 der medizinischen Fachgesellschaften und Dachverbände wurden mit der Prozedurenklassifikation CHOP 2011 umgesetzt. Damit die Kodierabteilungen der Spitäler die erbrachten Leistungen korrekt kodieren können, ist eine differenzierte ärztliche Dokumentation wichtig. Die FMH hat deshalb Dokumentationshilfen für komplexe und multimodale Kodes erarbeitet und den ärztlichen Fachgesellschaften zur Vernehmlassung vorgelegt. Die Dokumentationshilfen werden im ersten Quartal 2011 auf www.fmh.ch $\rightarrow$ Tarife $\rightarrow$ SwissDRG aufgeschaltet.

\section{Normativer Zuschlag für die Anlagenutzungskosten}

Gemäss Gesetz sind die Anlagenutzungskosten der Spitäler per 1. Januar 2012 in die Fallpauschalen des SwissDRG-Systems einzubeziehen. Eine Studie der KPMG [1] zeigte, dass der Entwicklungsstand der Anlagebuchhaltungen der Spitäler unterschiedlich und die Datenlage nicht ausreichend ist, um einen Zuschlag für die Anlagenutzungskosten kalkulieren zu können. Deshalb werden in einer dreijährigen Übergangslösung für die Abgeltung prozentuale, schweizweit einheitliche normative Zuschläge auf die Baserate festgelegt. Die Höhe des prozentualen Zuschlags wird zurzeit noch verhandelt.

\section{Tarifmodelle Psychiatrie und Rehabilitation}

In der Psychiatrie werden die beiden bestehenden Projekte «psysuisse» von H+ und «P-TAR» der Gesundheitsdirektion Zürich zusammengeführt und im Rahmen eines gemeinsamen Projekts vorangetrieben. Für die Rehabilitation wird das Projekt «STM Reha MTK» von $\mathrm{H}+$ und der MTK (Medizinal-Tarifkommission UVG) weitergeführt. Bei beiden Projekten fungiert der Verwaltungsrat der SwissDRG AG als Projektsteuerungsorgan. Die Ausarbeitung der beiden Tarifmodelle wird mehrere Jahre in Anspruch nehmen, d. h., die neuen Tarifmodelle stehen per 1. Januar 2012 noch nicht zur Verfügung.

\section{Qualitätssicherung: Ärzteschaft nicht vollwertig eingebunden}

Die SwissDRG AG hat die Qualitätskontrolle an den Nationalen Verein für Qualitätsentwicklung in Spitälern und Kliniken (ANQ) übertragen. Inzwischen haben die Träger des ANQ, H+, santésuisse, MTK und GDK, die Finanzierungsregelung ausgearbeitet. Vorgesehen sind folgende Messungen in der Akutsomatik: potentiell vermeidbare Rehospitalisationen und Reoperationen mit SQLape, nosokomiale Wundinfekte mit SwissNOSO, Prävalenzmessungen «Dekubitus» und «SturZ» mit dem Instrument LPZ (landesweite Prävalenzerhebung pflegebezogener Daten) und die Patientenzufriedenheit. Der Antrag der FMH an den ANQ auf Vollmitgliedschaft wurde abgelehnt.

\section{Weitere Zusatzentgelte werden erst für die SwissDRG-Version 2.0 geprüft}

Die FMH setzt sich seit langem dafür ein, dass im SwissDRG-System Zusatzentgelte etabliert werden. Diese sind für die leistungsgerechte Vergütung von teuren Medikamenten und Verfahren unerlässlich. In 
Deutschland haben die Zusatzentgelte wesentlich zur Güte des G-DRG-Systems beigetragen. Zurzeit führt der deutsche Fallpauschalenkatalog insgesamt 146 Zusatzentgelte auf, wovon 82 bewertet, d.h. mit einem Preis versehen sind. Die 64 unbewerteten Zusatzentgelte werden spitalindividuell verhandelt. In der Schweiz wird die SwissDRG-Einführungsversion 1.0 nur fünf [2] Zusatzentgelte aufweisen, obwohl die FMH beantragt hatte, jene Zusatzentgelte umzusetzen, für die ein Antrag besteht und mit denen in Deutschland gute Erfahrungen gesammelt wurden.
Tarifverhandlungen zur Festlegung der Baserate dürfen wir gespannt sein.

Die noch offenen Fragen bestätigen die Forderung der FMH, dass es während der Einführungsphase eine Übergangsregelung zur Abfederung von Systemverzerrungen braucht [4]. Finanzielle Garantien für die Spitäler während der Einführungsphase sind notwendig, damit diese nicht Verluste erleiden, nur weil ihr Behandlungsspektrum im SwissDRG-System ungenügend abgebildet ist. Nachdem die FMH mit ihrer Forderung lange Zeit alleine dastand, konnte sie

\section{Inzwischen konnte die FMH einige Partner von einer Übergangsregelung während der Einführungsphase überzeugen.}

Die SwissDRG AG hat zwar entschieden, die verbleibenden Anträge der Schweizer Fachgesellschaften zu prüfen, jedoch erst für die SwissDRG-Version 2.0. Die Einführungsversion 1.0 wird somit die im G-DRGSystem wichtigen Zusatzentgelte kaum berücksichtigen - dies obwohl sie wesentlich auf der deutschen Vorlage basiert und zudem zahlreiche (helvetisierte) deutsche Kostengewichte enthalten wird. Entsprechende Verzerrungen sind damit zu erwarten.

\section{Vergütung Frührehabilitation nun doch mit SwissDRG}

Die FMH konnte nach erfolgreicher Intervention bewirken, dass die Frührehabilitation bzw. die akuten und rehabilitativen Massnahmen nicht aus dem Swiss DRG-System ausgelagert, sondern mit der Einführungsversion 1.0 durch SwissDRG abgebildet und vergütet werden. Abgesehen davon wird die SwissDRG AG alle bisher noch unbewerteten DRGs der SwissDRG-Version 0.3 [3] für die Einführungsversion 1.0 nach einem definierten Verfahren bewerten.

\section{FMH setzt sich für Übergangsregelung ein}

Der zunehmende Zeitdruck dürfte sich zwar positiv auswirken, da die Partner ihre Kräfte bündeln und gemeinsam nach Kompromissen suchen müssen. Trotzdem werden bis zum 1. Januar 2012 nicht alle problematischen Punkte gelöst sein. SwissDRG ist ein lernendes System, und insbesondere die noch ungenügende Datenqualität lässt sich nicht kurzfristig auf einen Stichtag hin, sondern nur kontinuierlich verbessern. Zusätzlich zu den oben stehenden problematischen Punkten sind weitere wichtige Fragen, z.B. die Sicherung der Finanzierung der ärztlichen Weiterbildung, ebenfalls noch nicht abschliessend geklärt. Und auch auf die Ausgestaltung und den Verlauf der inzwischen einige Partner von diesem Ansatz überzeugen. Wermutstropfen ist, dass verschiedene Partner finanzielle Ausgleichsmechanismen nicht auf nationaler Ebene, sondern auf Ebene der Tarifverträge festlegen möchten. Da dies ungleich lange Spiesse schaffen würde, setzt sich die FMH weiterhin für eine national einheitliche Lösung ein.

\section{Literatur}

1 Vgl. www.swissdrg.org

2 Es handelt sich um folgende fünf Zusatzentgelte: drei bewertete Zusatzentgelte zu Nierenersatzverfahren, ein unbewertetes Zusatzentgelt für die Behandlung von Blutern mit Gerinnungsfaktoren und ein unbewertetes Zusatzentgelt «Implantatskosten Kunstherz».

3 Vgl. SwissDRG-Fallpauschalenkatalog Version 0.3, Anhänge 1 und 2.

4 Vgl. Meyer B. SwissDRG: ein Zwischenfazit. Schweiz Ärztezeitung. 2010;91(30/31):1127-8.

\section{Fortbildungsseminare}

Aufgrund der grossen Nachfrage bieten FMH und $\mathrm{H}+$-Bildung weitere Termine für spannende und informative Fortbildungsseminare an:

- Medizin und Ökonomie: Intensivseminar für Ärztinnen und Ärzte

- SwissDRG: Grundlagen für Ärztinnen und Ärzte

- Ärzte und Kodierung: Verantwortung unter SwissDRG

Details und Daten finden Sie auf www.fmh.ch $\rightarrow$ Tarife $\rightarrow$ SwissDRG

Die Seminare kommen ohne Sponsoring-Beiträge aus und sind nicht gewinnorientiert. 